\title{
Patient Concerns and Treatment Satisfaction in Patients Treated With Azelaic Acid Foam for Rosacea
}

\author{
Williamson T1; Cameron J1; McLeod K2; Turner B²; Quillen A2; LaRose A $^{1}$
}

Bayer HealthCare Pharmaceuticals Inc., Whippany, NJ, USA; ${ }^{2}$ Xcenda, Palm Harbor, FL, USA

\section{SYNOPSIS}

- Rosacea is a common, chronic, inflammatory skin disorder affecting the convexities of the central face and can be categorized into 4 main subtypes: erythematotelangiectatic, papulopustular, phymatous, and ocular. ${ }^{1,2}$

- Regardless of subtype, non-pharmacologic or behavioral interventions are useful for the management of skin flares; however, for patients with mild to moderate cases, especially of papulopustular rosacea, topical therapies are usually used as first-line therapy. ${ }^{1,3}$

- The use of topical medications, including metronidazole and azelaic acid gel, has shown efficacy in clinical trials vs placebo in reducing inflammatory lesion counts in patients with papulopustular rosacea; however, these treatments were associated with higher incidences of post-application skin discomfort, as patients reported burning, itching, and stinging sensations. ${ }^{1,2,4-6}$

- Formulations like azelaic acid foam have the potential to offer improvements over the side effect profiles of these treatment options.

\section{OBJECTIVE}

- This study aimed to survey patients with rosacea about their concerns, treatment satisfaction, and quality of life (QoL) associated with their azelaic acid foam treatment.

\section{METHODS}

\section{STUDY DESIGN}

- The study utilized a non-interventional, prospective, observational design and enrolled participants via email in collaboration with a patient support program, the Rosacea Concierge Program.

- A cross-sectional design was used to assess key patient concerns, treatment satisfaction, and $\mathrm{Q} o \mathrm{~L}$ related to azelaic acid foam for rosacea.

\section{SAMPLE SELECTION}

- 2,150 patients from the United States (US) who were enrolled in the Rosacea Concierge Program were invited to participate in the study.

- All inclusion and exclusion criteria were patient reported.

- Inclusion criteria:

\section{- At least 18 years of age}

- Diagnosis of rosacea by a medical professional

- Currently using azelaic acid foam as topical monotherapy for rosacea

- Willing and able to provide voluntary, informed consent to participate in the study

- Exclusion criteria:

- Use of any other topical treatment for rosacea at the time of enrollment

\section{STUDY ENDPOINTS}

- Eligible, consenting patients completed a 1-time survey assessing demographics, clinical characteristics (ie, rosacea-relevant comorbidities and complications), treatment history, and adverse events.

- Table 1 includes a brief overview of the 3 questionnaires included in the survey.

\begin{tabular}{|c|c|}
\hline Questionnaire & Details \\
\hline $\begin{array}{l}\text { Rosacea Treatment } \\
\text { Preference } \\
\text { Questionnaire }\end{array}$ & $\begin{array}{l}\text { - 9-question survey composed of both aided and unaided } \\
\text { questions. } \\
\text { - Assesses patient self-reported rosacea subtype and severity } \\
\text { and evaluates drug characteristics that contribute to patient } \\
\text { satisfaction/dissatisfaction and treatment decisions with } \\
\text { rosacea topical treatments. } \\
\text { - Respondents list up to } 5 \text { concerns as well as up to } 5 \text { side effects } \\
\text { with their current topical rosacea treatment experienced in } \\
\text { the past } 4 \text { weeks and rate the importance of each reported } \\
\text { concern or side effect. } \\
\text { - Respondents rank a list of pre-identified issues with topical } \\
\text { rosacea treatment (eg, efficacy, cost, texture, dryness, etc) on a } \\
\text { scale of importance from } 0 \text { to } 10 \text { (with } 0=\text { not at all important; } \\
10=\text { extremely important) in terms of how important the issue } \\
\text { is when they consider using a new topical rosacea treatment. }\end{array}$ \\
\hline SATMED-Q & $\begin{array}{l}\text { 17-question, validated, multidimensional, generic questionnaire } \\
\text { designed for use in patients with any chronic disease treated with } \\
\text { medicines measuring treatment satisfaction. } \\
\text { - Composed of } 6 \text { domains: } \\
\text { - Undesirable side effects (3 questions) } \\
\text { - Efficacy ( } 3 \text { questions) } \\
\text { - Convenience and ease of use (3 questions) } \\
\text { - Impact of medicine ( } 3 \text { questions) } \\
\text { - Medical follow-up/review (2 questions) } \\
\text { - Overall opinion (3 questions) }\end{array}$ \\
\hline DLQI & $\begin{array}{l}\text { - } 10 \text {-question, widely used dermatology-related QoL tool. } \\
\text { Questions are general and cover symptoms and feelings, daily } \\
\text { activities, leisure, work and school, personal relationships, and } \\
\text { treatment experience over the previous week. }\end{array}$ \\
\hline
\end{tabular}

STATISTICAL ANALYSIS

- All study analyses conducted were exploratory and descriptive in nature.

- The primary analysis population set included all patients who met the eligibility criteria and completed the survey.

- Baseline characteristics were calculated as mean values for continuous variables and percentages for categorical variables.

- Proportions of patients listing each concern or side effect related to azelaic acid foam in the Rosacea Treatment Preference Questionnaire were assessed.

- All the importance or tolerability scores that patients assigned to each concern or side effect, the satisfaction score from the Satisfaction with Medicines Questionnaire (SATMED-Q), and the QoL score from the Dermatology Life Quality Index (DLQI) were computed and summarized using means standard deviations and medians as appropriate.

- As an exploratory analysis to assess the association between concerns and side effects vs overall treatment satisfaction and overall QoL, regression analyses were conducted. 


\title{
Patient Concerns and Treatment Satisfaction in Patients Treated With Azelaic Acid Foam for Rosacea
}

\author{
Williamson T1; Cameron J1; McLeod K²; Turner B²; Quillen A $^{2}$; LaRose A $^{1}$
}

Bayer HealthCare Pharmaceuticals Inc., Whippany, NJ, USA; ${ }^{2}$ Xcenda, Palm Harbor, FL, USA

\section{RESULTS}

\section{PATIENT ATTRITION}

- Study recruitment and patient attrition are summarized in Figure 1.

- 2,150 program-identified patients were invited to participate, 150 patients responded, and 54 met all eligibility criteria and were included in the study

Figure 1. Study Recruitment and Patient Attrition

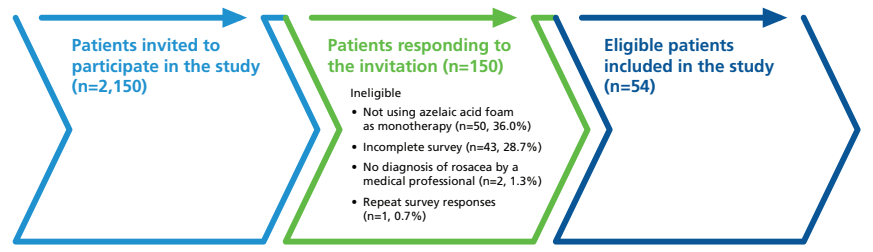

\section{DEMOGRAPHICS}

- A total of 54 patients were included in the study. Patient population characteristics and rosacea medical history are described in Table 2.

- Participants were primarily female $(90.7 \%)$, ranging in age from 26 to 63 years.

- The majority of participants $(77.8 \%)$ reported no rosacea-relevant medical conditions.

Table 2. Baseline Characteristics and Rosacea-relevant Medical Conditions

\begin{tabular}{|c|c|c|c|}
\hline \multirow{3}{*}{ Gender, n (\%) } & \multirow[b]{2}{*}{ Female } & \multicolumn{2}{|c|}{ Total, $\mathrm{N}=54$} \\
\hline & & 49 & 90.7 \\
\hline & Male & 5 & 9.3 \\
\hline \multirow{4}{*}{ Age (years) } & Mean (standard deviation) & 48.1 & (9.4) \\
\hline & Min & 26.0 & - \\
\hline & Median & 48.5 & - \\
\hline & Max & 63.0 & - \\
\hline \multirow{7}{*}{$\begin{array}{l}\text { Health insurance } \\
\text { coverage type, } n \\
(\%)\end{array}$} & Preferred provider organization & 41 & 75.9 \\
\hline & Health maintenance organization & 8 & 14.8 \\
\hline & $\begin{array}{l}\text { Worker's compensation/motor } \\
\text { vehicle/third-party liability }\end{array}$ & 0 & 0.0 \\
\hline & Medicaid & 2 & 3.7 \\
\hline & Medicare/Medicare supplemental & 0 & 0.0 \\
\hline & Indemnity & 0 & 0.0 \\
\hline & Other & 5 & 9.3 \\
\hline \multirow{6}{*}{$\begin{array}{l}\text { Rosacea-relevant } \\
\text { medical conditions, } \\
\text { n (\%) }\end{array}$} & None & 42 & 77.8 \\
\hline & Depression & 5 & 9.3 \\
\hline & Migraine & 5 & 9.3 \\
\hline & Conjunctivitis & 4 & 7.4 \\
\hline & Blepharitis & 1 & 1.9 \\
\hline & Corneal neovascularization/keratitis & 0 & 0 \\
\hline
\end{tabular}

- The most common subtypes reported by study participants were erythematotelangiectatic and papulopustular $(74.1 \%$ each), with $59.3 \%$ of participants reporting "mild" rosacea symptoms (16.7\% "absent"; $24.1 \%$ "moderate") in the 4 weeks before enrollment.

- Only $13.0 \%$ of patients reported no previous rosacea treatment.
- The most commonly reported topical agent for prior rosacea treatment was metronidazole gel (7.4\%).

\section{PATIENT CONCERNS}

- The majority of patients reported no concerns (74.1\%) with their treatment (Figure 2). The biggest concern reported was cost (11.1\% of patients), with a mean importance score (IS) on a 10-point scale of 9.3 (Figure 3).

Figure 2. Patient Concerns With Rosacea Treatment

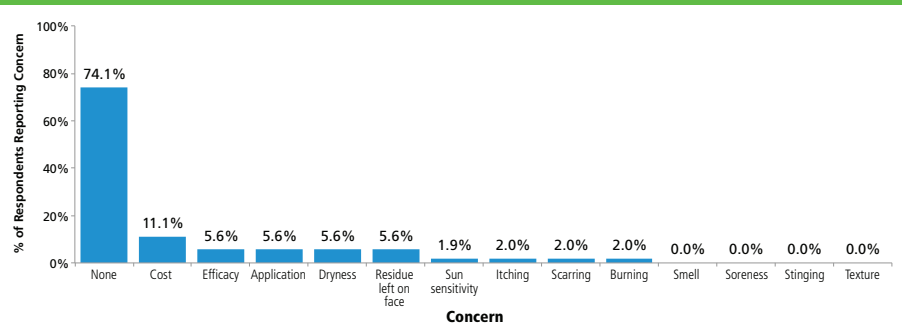

Figure 3. Rosacea Treatment Concerns' Mean Importance Scores

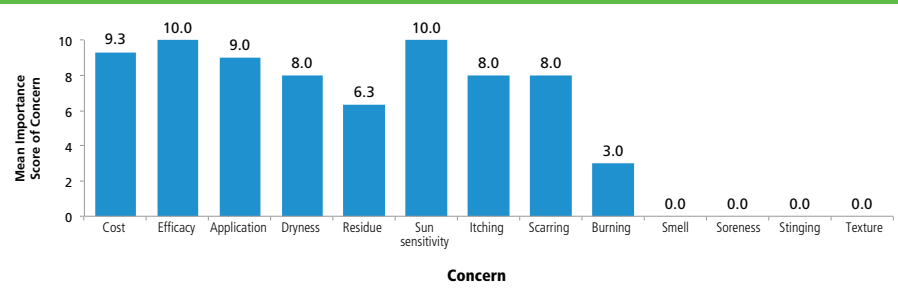

- A majority (77.8\%) of patients reported no side effects (Figure 4). Dryness was the most commonly reported side effect $(13.0 \%$; IS: 5.3$)$. Other side effects reported included stinging $(7.4 \%$, IS: 2.5$)$, itching $(5.6 \%$; IS: 4.7$)$, redness $(5.6 \%$; IS: 8.3 ), and burning (3.7\%; IS: 7.0) (Figure 5).

Figure 4. Side Effects With Rosacea Treatment

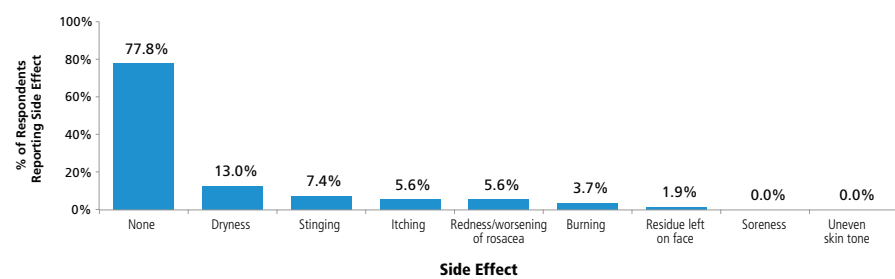

Figure 5. Mean Importance Scores of Rosacea Treatment Side Effects

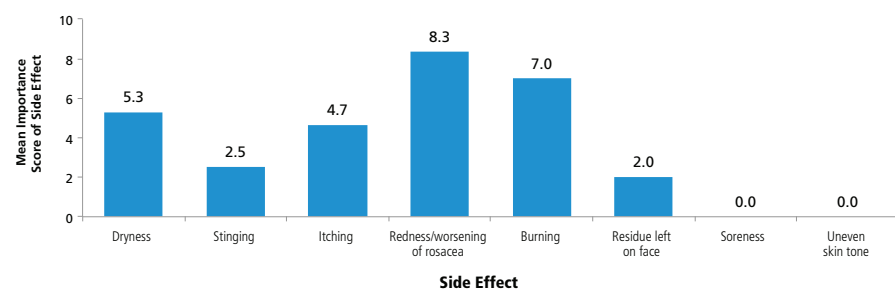




\title{
Patient Concerns and Treatment Satisfaction in Patients Treated With Azelaic Acid Foam for Rosacea
}

\author{
Williamson T1; Cameron J1; McLeod K²; Turner B²; Quillen A $^{2}$; LaRose A $^{1}$
}

Bayer HealthCare Pharmaceuticals Inc., Whippany, NJ, USA; ${ }^{2}$ Xcenda, Palm Harbor, FL, USA

\section{TREATMENT SATISFACTION AND QOL}

- The global satisfaction (SATMED-Q) mean score was 79.0 and treatment effectiveness meanscore was 70.8(Figure 6). Standardized scores for the SATMED-Q ranged from 0 to 100, with an overall score of 59.3 indicating feeling neutral and each additional 13.4-point increase indicating a clinically meaningful movement toward satisfaction.

- The impact of rosacea on QoL was "minimal" (mean DLQI score: 2.35). DLQI scores ranged from 0 to 30 (with 0-1 indicating rosacea has no effect on QoL and 21-30 indicating rosacea has an extremely large effect on QoL).

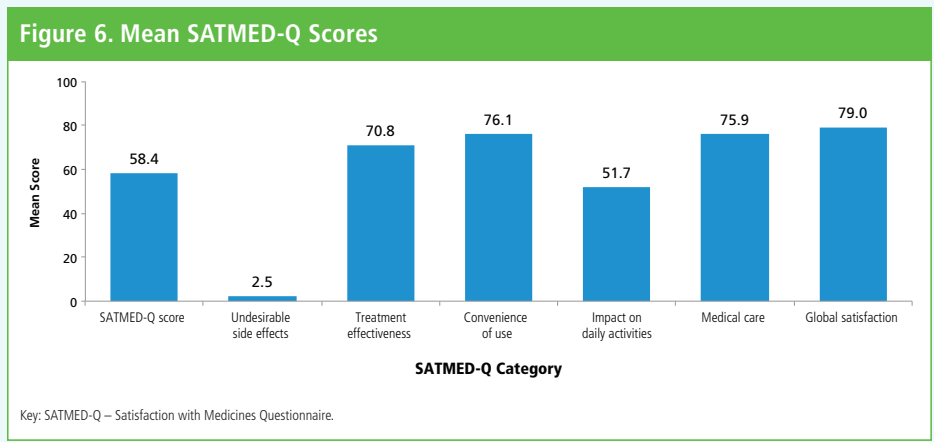

\section{EXPLORATORY ANALYSIS}

- In regression models used for the exploratory analysis, increasing dryness importance scores were significantly associated with worsening treatment satisfaction and QoL in SATMED-Q and DLQI.

\section{LIMITATIONS}

- Due to the limited respondent pool, further research is needed to confirm these results.

- The International Society of Pharmacoeconomics and Outcomes Research (ISPOR) recommends that a minimum sample size of 200 patients is needed to obtain meaningful survey results in research on patient-reported outcomes. A total of 2,150 patients were invited to participate in this study, and 150 responded; however, only 54 met eligibility criteria and were enrolled in the study.

\section{CONCLUSION}

- Azelaic acid foam was well tolerated and efficacious, with less than $26 \%$ of participants reporting any concerns or side effects and $6 \%$ reporting a concern with treatment efficacy.

- Azelaic acid foam users reported favorable results in the domains of burning, itching, and stinging.

- Due to the limited respondent pool, further research is needed to confirm these results.

\section{REFERENCES}

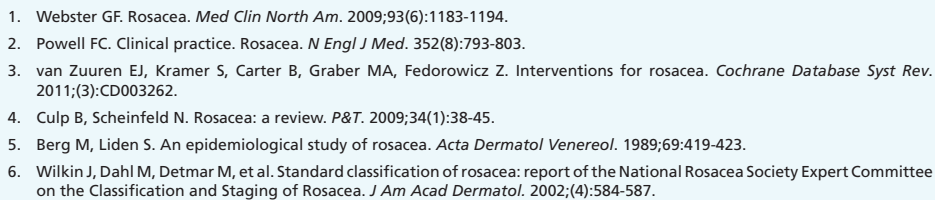

